\title{
4 Betriebliche Beteiligung in der Umsetzung
}

In Abschnitt 2.2 wurden die Merkmale des deutschen Modells der Arbeitsbeziehungen vorgestellt und die rechtlichen Grundlagen zur Einbindung und Arbeitsweise von Betriebsräten skizziert. Abschnitt 3.3 stellte die in Analogie dazu entstandene betriebliche Interessenvertretung in WfbM und die verordnungsrechtlichen Befugnisse von Werkstatträten vor. Zunächst die gesetzliche Ausrichtung der Arbeit von Betriebsräten zu behandeln und anschließend die Vorgaben zur Arbeit der Werkstatträte zu fokussieren war erforderlich, da die WMVO in Anlehnung an die Regelungen des BetrVG entstanden ist (Cramer, 2009, S. 387) und sich die Vorgaben so besser nachvollziehen lassen.

Nun stellt sich die Frage, wie sich die Rechtsnormen in der Umsetzung ausgestalten. Wie wird betriebliche Mitbestimmung lebensweltlich erlebt? Wie fällt die Arbeit der Gremien jenseits der formalen Anlagen de facto aus? Gelingt es ihnen, ihre Rechte zu mobilisieren, und kann damit eine Einflussnahme im Betrieb realisiert werden? Was zeichnet erfolgreiche Gremien aus? Wie in der Einleitung dieser Arbeit bereits erwähnt, liegen zu Werkstatträten bisher kaum empirische Ergebnisse vor. Ersatzweise lässt sich die betriebliche Mitbestimmungsforschung konsultieren, um Antworten auf die aufgeworfenen Fragen - zumindest für Betriebsräte $-\mathrm{zu}$ finden. Dazu werden die betriebliche Mitbestimmungsforschung und vorliegende Ergebnisse in Abschnitt 4.1 thematisiert. Den vorliegenden Kenntnisstand über die Arbeit von Werkstatträten in WfbM greift anschließend Abschnitt 4.2 auf. Abgerundet wird das Kapitel mit einem Exkurs in die Rechtssoziologie (Abschnitt 4.3). Hiermit lässt sich vertiefen, was allgemein über die Inanspruchnahme von Rechtsansprüchen angenommen wird und wie Rechtsmobilisierung vonstattengehen kann. Am Ende wird wieder ein Zwischenfazit über die Ergebnisse des Kapitels gezogen (Abschnitt 4.4).

\subsection{Erkenntnisse aus der betrieblichen Mitbestimmungsforschung}

Um betriebliche Mitbestimmungsforschung zu charakterisieren, wird in Abschnitt 4.1.1 ein kleiner Einblick in das Forschungsfeld, zu übergreifenden Ergebnissen und zu vorhandenen sozialempirischen Arbeiten gegeben. Anschließend werden vier Forschungsarbeiten zu Betriebsräten genauer vorgestellt (Abschnitt 4.1.2). Hierbei liegt das Ziel nicht nur darin, exemplarische Themen und Erkenntnisse zu erörtern, sondern auch herauszuarbeiten, wie die Studien methodisch umgesetzt werden. Dies wird für die vorliegende Arbeit als wichtig erachtet: zum einen, da empirische Erhebungsergebnisse nur im Zusammenhang mit ihrer methodischen 
Erarbeitung beurteilt werden können. Zum anderen diente die Betrachtung der Vorgehensweisen dazu, das eigene Vorhaben methodisch adäquat vorzubereiten. Um den Lesefluss flüssig zu halten, sind die methodischen Hinweise teilweise in Fußnoten gesetzt.

\subsubsection{Einblick in die empirische Mitbestimmungsforschung}

Der Forschungsstrang der betrieblichen Mitbestimmung kann auf eine breite empirische Basis verweisen. Beheimatet in der Industrie- und Betriebssoziologie umfasst der Forschungsstand unterschiedliche disziplinäre Zugänge und Bezüge. Es liegen u. a. sozial-, rechts- und wirtschaftswissenschaftliche Studien vor (Diefenbacher \& Nutzinger, 1980; Kißler et al., 2011, S. 151). Die Themen umfassen die Mitbestimmung als „Idee und Wirklichkeit“ (Greifenstein \& Kißler, 2010, S. 12), sprich, das Themenspektrum bewegt sich zwischen ideologischen Erörterungen und tatsachenorientierten Studien. Als klassische Forschungsgegenstände der sozialempirischen Arbeiten werden für Deutschland der Aufsichts-, der Betriebs-/Personalrat sowie die Arbeitsgruppe klassifiziert. Dies resultiert aus den drei Umsetzungsebenen der Mitbestimmung (Unternehmen, Betrieb, Arbeitsplatz, siehe Abschnitt 2.2) und einer institutionszentrierten Tatsachenorientierung der Industrie-/Betriebssoziologie (Müller-Jentsch, 2007). Der Überblicksband „Mitbestimmung im Spiegel der Forschung. Eine Bilanz der empirischen Untersuchungen 1952-2010“ (Greifenstein \& Kißler, 2010) zeigt den Fundus der vorliegenden Arbeiten auf. Alleine für den Zeitraum 1990-2010 werden 165 empirische Ersterhebungen bzw. Langzeitstudien gelistet, die den Ansprüchen empirischer Sozialforschung genügen (ebd., S. 29). Die gute Studienlage gilt jedoch lediglich für den privatwirtschaftlichen Sektor. Für den öffentlichen Dienst und den kirchlichen Bereich (siehe Abschnitt 2.2.2) bestehen nur wenige Studien (Brehmer, 2014, S. 2). Insbesondere die Umsetzung des konfessionellen Mitarbeitervertretungsrechts gilt als empirisch kaum untersucht (Ensinger, 2006, S. VII; Jakobi, 2007, S. 20).

Zudem muss mitgedacht werden, dass sich die Mitbestimmungsforschung durch vorhandene Reglementierungen, branchenspezifische Gegebenheiten, gewerkschaftliche Zugehörigkeiten und ihre Finanzierungsquellen auf bestimmte Sektoren fokussiert. ${ }^{44}$ Nach den bereits erwähnten Zahlen des IAB-Betriebspanels 2018 gibt es derzeit nur in $9 \%$ der

\footnotetext{
${ }^{44}$ Die Forschungsfinanzierung ist stark durch die Hans-Böckler-Stiftung geprägt. Weitere Finanzierungsstellen sind u. a. die Deutsche Forschungsgemeinschaft, der Deutsche Gewerkschaftsbund und die Arbeitgeberverbände. Ferner liegen eine Reihe von Qualifizierungsarbeiten vor (Greifenstein \& Kißler, 2010, S. 186 ff.).
} 
privatwirtschaftlichen Betriebe ab fünf Beschäftigten (ohne Landwirtschaft) überhaupt einen Betriebsrat (siehe zum Panel Fußnote 12). Der Verbreitungsgrad steigt mit zunehmender Betriebsgröße und variiert stark branchenspezifisch. Während $87 \%$ aller Betriebe mit 500 oder mehr Beschäftigten in Westdeutschland einen Betriebsrat haben (in Ostdeutschland $88 \%$ ), trifft dies lediglich auf $5 \%$ der Betriebe mit 5-50 Beschäftigten zu (6\% in Ostdeutschland). Die Gremien sind mit einer Einrichtungsquote von 40 \% am häufigsten im Bereich „Energie/Wasser/Abfall/Bergbau“ verbreitet, was sich traditionell begründet. Im „Baugewerbe“ und „Gaststätten- und sonstigem Dienstleistungsbereich“ sind sie hingegen kaum anzutreffen und nur bei $3 \%$ (Westdeutschland) bzw. $2 \%$ (Ostdeutschland) der Betriebe eingerichtet (Ellguth \& Kohaut, 2019, S. 295). Neben der Branche und der Betriebsgröße sind personenbezogene Wissensbestände über die gesetzlichen Möglichkeiten und Haltungen gegenüber betrieblicher Mitbestimmung wesentlich, die mit darüber entscheiden, ob ein Betriebsrat errichtet wird und wie dessen Arbeit ausfällt. Im Folgenden werden hierzu übergreifende Ergebnisse aus der erwähnten Forschungsbilanz von Greifenstein und Kißler (2010) vorgestellt.

\section{Ergebnisse zu personenbezogenen Wissensbeständen und Haltungen bei Beschäftigten}

Die empirischen Ergebnisse stimmen dahingehend überein, dass das Wissen über die bestehenden Mitbestimmungsmöglichkeiten bei Arbeitnehmer*innen und anderen Beschäftigten generell mangelhaft ausfällt. Für den individuellen Wissensstand spielen die betriebliche Position und die Arbeitssituation eine wesentliche Rolle. Das Wissen nimmt u. a. mit der Dauer der Betriebszugehörigkeit, mit gewerkschaftlichen Kontakten, dem Grad der Qualifikation und arbeitsplatznahen Mitbestimmungserfahrungen zu. Demgegenüber hemmen restriktive Arbeitsbedingungen das Mitbestimmungswissen und die Beteiligungsbereitschaft. Eine hohe Arbeitsplatzzufriedenheit und größere Einflussmöglichkeiten wirken sich wiederum positiv auf die Einstellung zur Mitbestimmung aus. Empirisch zeigt sich, dass die mit dem Beteiligungsinstrument des Betriebsrats verbundenen Gestaltungsmöglichkeiten im Erfahrungshorizont der Beschäftigten erst relevant werden, sobald die primären Interessenlagen wie etwa Lohn- und Beschäftigungssicherheit gegeben sind (Greifenstein \& Kißler, 2010, S. 45-50). Die Errichtung eines Betriebsrats ist folglich bereits voraussetzungsvoll. Doch welche Erkenntnisse liegen nun dazu vor, wenn das Gremium tätig wird? 


\section{Vorliegende Forschungsarbeiten zu Betriebsräten}

Zur Arbeit von Betriebsräten werden drei thematische Bereiche klassifiziert:

- Ein grundlegender erster Studienstrang richtet sich auf die innerbetriebliche Position von Betriebsräten. Aus diesen Studienergebnissen geht hervor, dass die Wahrnehmung und Ausgestaltung der gegebenen gesetzlichen Beteiligungsrechte stark variiert und erheblich durch die innerbetriebliche Stellung des Betriebsrats und die sozialen Beziehungen im Betrieb beeinflusst wird (Greifenstein \& Kißler, 2010, S. 57 f.). Neben der Art des Beziehungsverhältnisses zwischen Betriebsrat und Geschäftsleitung gilt für die eingenommene Position des Gremiums der Mobilisierungsgrad der Belegschaft als wesentlich. Dieser äußert sich etwa in Form der gewerkschaftlichen Affinität und der Konfliktbereitschaft der Beschäftigten. Als Beispiel einer Arbeit, die sich auf die eingenommene Rolle der Interessenvertretung richtet, wird im folgenden Abschnitt die Betriebsratstypologie von Kotthoff (1981) vorgestellt.

- Ein zweiter Bereich von Forschungsarbeiten handelt davon, wie Betriebsratsarbeit ausgestaltet wird und womit die Ausgestaltung zusammenhängt. Hier zeigt sich bspw., dass die Unternehmensgröße entscheidend beeinflusst, wie die Arbeitsressourcen und -ergebnisse eines Betriebsrats ausfallen. Im Hinblick auf die Mitbestimmungsbereiche wird u. a. konstatiert, dass die Beteiligungsrechte in sozialen Angelegenheiten ausgeprägter sind als bei den personellen Angelegenheiten und dass sich Abschläge bei der Funktionsweise des Wirtschaftsausschusses zeigen (Greifenstein \& Kißler, 2010, S. 59 f.).

- Als dritter Bereich finden sich Studien, die von den neuen Themen der Mitbestimmung handeln. Hiermit sind veränderte rechtliche Grundlagen, neue Technologien und andere gewandelte Arbeitsmarktanforderungen gemeint. Festgehalten wird in diesen Arbeiten bspw. ein zunächst defensives Verhalten der Gremien bei Arbeitsgestaltungsprozessen, was mit einem vorliegenden Überforderungs-/Kompetenzproblem begründet wird (ebd., S. 63). Die Forschung konnte aber auch herausarbeiten, dass ein neuer Betriebsratstyp entsteht, dem es als „Wissensmanager“ (ebd., S. 106) gelingt, Innovationsprozesse voranzutreiben.

Um diese kursorische Studienübersicht zu vertiefen, werden im nächsten Abschnitt vier ausgewählte Arbeiten zu Betriebsräten genauer vorgestellt. Zunächst wird auf die qualitative Forschungsarbeit von Kotthoff (1981) als „Klassiker“ unter den Studien eingegangen. Anschließend werden drei quantitative Studien präsentiert, die im Hinblick auf die Gremienarbeit von Werkstatträten als relevant erscheinen. So generiert die Arbeit von Müller-Jentsch, Malinowsi, 
Seitz und Drescher (1998) grundlegendes Wissen zur Betriebsratsarbeit in einem spezifischen Sektor und typisiert quantitativ die eingenommene Rolle im Betrieb. Als Vertiefung dieser Betriebsrätetypologie richten Minssen und Riese (2007) den Blick auf die interne Arbeitsorganisation und die Einflussnahme von Betriebsräten bei Umstrukturierungsprozessen. Die Arbeit von Nienhüser (2005) richtet sich demgegenüber noch spezifischer aus und erforscht, inwieweit abgeschlossene Betriebsvereinbarungen mit der Einschätzung von Betriebsräten als stark und kooperativ zusammenhängen.

\subsubsection{Ausgewählte Studien und deren Ergebnisse}

\section{Der Klassiker unter den Betriebsrätetypologien}

In einer empirisch-qualitativen soziologischen Arbeit von 1981 spürte Kotthoff den eingenommenen Rollen von Betriebsräten nach, die in verschiedenen betrieblichen Herrschafts-/Beziehungsgefügen entstehen. Das Sample seiner Studie umfasste 63 Betriebe im südbadischen Raum. ${ }^{45}$ In seiner Ergebnisdarstellung arbeitete Kotthoff sechs verschiedene Betriebsratstypen heraus (siehe Tabelle 4). Ergänzt wurde die Typologie um den fiktiven „klassenkämpferischen Betriebsrat“, der im Sample nicht anzutreffen war, auf Basis anderer Studien in seltenen Fällen jedoch vermutet wurde. Zu beachten ist, dass der Autor die Klassifizierungen als Prototypen beschreibt, die das ,charakteristisch Erscheinende deutlich repräsentieren“ (ebd., S. 244).

In zwei Drittel der untersuchten Betriebe war eine defizitäre Interessenvertretung (Typ I bis IV) gegeben. Allerdings arbeitete Kotthoff in einer Folgeuntersuchung in den identischen Betrieben in den 1990er-Jahren heraus, dass ein Wandel zugunsten der vertretungswirksamen Typen stattgefunden hatte. Die defizitäre Interessenvertretung war nunmehr in einem Drittel der Betriebe vorhanden (Kißler et al., 2011, S. 113). Im Ergebnis der Studie unterscheidet

${ }^{45}$ Diese wurden aus Karteien der klassischen Gewerkschaften (IG Metall, Chemie, Textil etc.) gezogen. Das erzeugte Material (primär leitfadengestützte Interviews mit Betriebsratsmitgliedern und der Geschäftsleitung) wurde zunächst statistisch betrachtet, um die Fragekomplexe (u. a. Haltungen gegenüber dem Betriebsrat bzw. dem BetrVG, Deutung der eingenommenen Position) darzustellen und vergleichende Indikatoren zu erzeugen. Anschließend wurde das Material inhaltsanalytisch ausgewertet, verdichtet und in Form einer Typologie mit den Betrieben als Falleinheit kontrastiert. Die Typenbildung erfolgte u. a. anhand der (wahrgenommenen) innerbetrieblichen Stellung und der Funktion des Betriebsrates, der angewandten Mitbestimmungskommunikation und der Strategien der Gremien (Kotthoff, 1981, S. 37 ff.). 
Kotthoff die defizitäre von der vertretungswirksamen Partizipation, wobei er Partizipation als Synonym für Mitbestimmung verwendet.

Tabelle 4: Betriebsratstypen nach Kotthoff (1981)

\begin{tabular}{|c|c|}
\hline Betriebsratstyp & Beschreibung \\
\hline $\begin{array}{l}\text { I: Der ignorierte } \\
\text { Betriebsrat }\end{array}$ & $\begin{array}{l}\text { - Ist bedeutungslos, etwas Nebensächliches. } \\
\text { - Formale Abläufe des BetrVG (Sitzungen etc.) finden kaum statt. } \\
\text { - Erhält keinerlei Informationen, Beteiligungsrechte werden igno- } \\
\text { riert und auch gar nicht angestrebt. }\end{array}$ \\
\hline $\begin{array}{l}\text { II: Der isolierte } \\
\text { Betriebsrat }\end{array}$ & $\begin{array}{l}\text { - Dient als formale Legitimation der Geschäftsführung, deren } \\
\text { Willkür er unterliegt. } \\
\text { - Formale Arbeitsmittel sind gegeben, eine betriebliche Einfluss- } \\
\text { nahme wird verweigert. }\end{array}$ \\
\hline $\begin{array}{l}\text { III: Der Betriebsrat } \\
\text { als Organ der } \\
\text { Geschäftsleitung }\end{array}$ & $\begin{array}{l}\text { - Die Betriebsratsmitglieder nehmen im Betrieb eine privilegierte } \\
\text { Stellung ein. } \\
\text { - Die Handlungen bestehen darin, die Vorstellungen und Wünsche } \\
\text { der Geschäftsführung durchzusetzen. }\end{array}$ \\
\hline $\begin{array}{l}\text { IV: Der respek- } \\
\text { tierte zwiespältige } \\
\text { Betriebsrat als } \\
\text { Ordnungsfaktor }\end{array}$ & $\begin{array}{l}\text { - Formale Abläufe des BetrVG werden eingehalten, eine ernst- } \\
\text { hafte Einflussnahme wird durch (verborgene) Absprachen zwi- } \\
\text { schen Geschäftsführung und Betriebsratsspitze unterwandert. } \\
\text { - Die Betriebsratsspitze begreift sich als Vermittler. }\end{array}$ \\
\hline $\begin{array}{l}\text { V: Der respektierte } \\
\text { standfeste } \\
\text { Betriebsrat }\end{array}$ & $\begin{array}{l}\text { - Selbstverständnis als Interessenvertretung der Arbeitnehmer. } \\
\text { - Handelt vertretungswirksam mit enger Orientierung an den } \\
\text { rechtlichen Rahmen, trägt Konflikte offen aus. }\end{array}$ \\
\hline $\begin{array}{l}\text { VI: Der Betriebsrat } \\
\text { als kooperative } \\
\text { Gegenmacht }\end{array}$ & $\begin{array}{l}\text { - Intensive Kommunikation mit der Belegschaft, stabile gremien- } \\
\text { interne Zusammenarbeit, politisches Selbstverständnis als Inte- } \\
\text { ressenvertretung. } \\
\text { - Zur Geschäftsführung besteht eine konflikthafte, „,stabile Ver- } \\
\text { trauensbeziehung“(S. 241), eigeninitiativ handelnd, kann er ef- } \\
\text { fektiv Einfluss ausüben. }\end{array}$ \\
\hline $\begin{array}{l}\text { Ergänzung: Der } \\
\text { klassenkämpferi- } \\
\text { sche Betriebsrat }\end{array}$ & $\begin{array}{l}\text { - Erlebt den gesetzlichen Rahmen des BetrVG als Begrenzung. } \\
\text { - Hält sich nicht an die Vorgaben, greift zu eigenen/kämpferischen } \\
\text { Mitteln, um seine Interessen durchzusetzen. }\end{array}$ \\
\hline
\end{tabular}

Anmerkung. Eigene Anfertigung nach Kotthoff (1981, S. 46-245). 
Die wichtigste Bedingung für eine vertretungswirksame Partizipation (Typ V und VI) sei zunächst, dass diese gewollt werde. Die Initiative zur Partizipation geht dabei nicht von oben, sondern von der Belegschaft und dem Betriebsrat aus. Die defizitäre Vertretung ist i. d. R. dort anzutreffen, wo der Partizipationswille bei den Betriebsräten nicht vorhanden ist und die gegebenen Verhältnisse als nicht veränderbar gedeutet werden. Die eigene lokale Betriebswelt kann wiederum nur als änderungsbedürftig und veränderbar erscheinen, wenn ein äußerer Bezugspunkt vorliegt, bspw. in Form eines Vergleichs mit anderen Betrieben oder einer Weltanschauung (Kotthoff, 1981, S. 258). Neben dem grundlegenden Partizipationswillen wird eine weitere fundamentale Komponente für betriebsrätliche Vertretungsmacht in einer interessierten und informierten Belegschaft gesehen. Laut Kotthoff ist diese jedoch nur selten gegeben, was er u. a. damit begründet, dass in der Belegschaft das Qualifizierungsniveau oft kein akademisches sei und eine größere Eigeninitiative zum Wissensausgleich notwendig ist.

Eine weitere Gelingensbedingung für vertretungswirksame Arbeit bildet nach den Studienergebnissen der Erwerb von Sach- und Fachkompetenz und von taktischem Geschick in den Detailfragen der Betriebsratsarbeit. Schwierig ist nach Kotthoff hieran, dass dazu ein Arbeitseinsatz notwendig ist, der nur mit einer Freistellung erreicht werden kann, wodurch gremienintern oftmals eine Hierarchisierung zwischen Betriebsratsspitze und Mitgliedern resultiere. Die Spitze kann wiederum dazu neigen, gegenüber der Geschäftsführung in kooperative Muster zu verfallen. Um diesen Tendenzen zu begegnen, gelte es, alle Gremienmitglieder in die Arbeit einzubinden und diesen die Kontrolle über die Betriebsratsspitze zu bewahren.

Überraschend positiv ist abschließend der Befund, dass Geschäftsleitungen mit den konfliktbereiten Betriebsräten (Typ V und VI) genauso zufrieden sind wie diejenigen, die das Gremium als verlängerten Arm der Geschäftsleitung (Typ III) verwenden (ebd., S. 260 ff.).

\section{Übernahme neuer Aufgaben- und Verantwortungsgebiete}

Eine quantitative Typologie zur Annahme neuer Aufgaben von Betriebsräten liegt aus einer Befragung von rund 730 Betrieben im Maschinen- und Anlagenbau aus dem Jahr 1996 vor (Müller-Jentsch et al., 1998; Müller-Jentsch \& Seitz, 1998). Die Fragestellungen des Forschungsprojekts richteten sich darauf, Erkenntnisse über veränderte Aufgaben, innerbetriebliche Arbeitsbeziehungen und außerbetriebliche Einbindungen zu erhalten. Die Erhebungsinhalte umfassten u. a. die Ausstattung der Betriebsräte, die aktuelle Betriebsratspraxis, die Beziehung zur Betriebsleitung (mit Fragen zu Verfahren vor Einigungsstellen und Arbeitsgerichten), Kontakte zu Gewerkschaften, betriebliche neue Arbeitsformen (z. B. Qualitätszirkel, 
Gruppenarbeit), abgeschlossene Betriebsvereinbarungen und Arbeitszeitmodelle (MüllerJentsch et al., 1998, Anhang 1). ${ }^{46}$ Der empirische Ergebnisbericht enthält eine Typologie, die Betriebsräte anhand der Auseinandersetzung mit neuen Themen (etwa neue Technologien, neue Arbeitsformen oder gewandelte Arbeitsmarktanforderungen) klassifiziert. Analysebasis ist ein Index, der aus bis zu 24 Punkten bestehen kann. ${ }^{47}$ Erreicht wurden von den befragten Gremien maximal 18 Punkte. Tabelle 5 zeigt den jeweils erreichten Summenscore, mit dem auch die Typeneinteilung vorgenommen wurde.

Tabelle 5: Betriebsratstypologie von Müller-Jentsch, Malinowsi, Seitz und Drescher (1998)

\begin{tabular}{llll}
\hline Betriebsratstyp & Punkte & $n$ & In Prozent \\
\hline I: Konventioneller bzw. formeller Betriebsrat & $0-1$ & 219 & $30 \%$ \\
II: Engagierter Betriebsrat & $2-5$ & 313 & $43 \%$ \\
III: Ambitionierter Betriebsrat & $6-9$ & 137 & $19 \%$ \\
IV: Co-Manager & $10-18$ & 53 & $7 \%$ \\
\hline
\end{tabular}

Anmerkung. Eigene Anfertigung nach Müller-Jentsch et al. (1998, S. 85).

Der Indexkonstruktion entsprechend umfasst Typ I Gremien, bei deren Arbeitsinhalten neue Arbeitsthemen noch nicht auftauchten. Diese Betriebsräte bleiben ,,auf den traditionellen Aufgabenbereich beschränkt“ (ebd., S. 83). Allerdings geht aus der Ergebnisdarstellung nicht hervor, inwieweit in den anderen Themenbereichen, wie etwa Arbeitsschutz oder Personalfragen (ebd., Anhang 1, S. 19 f.), überhaupt eine Aufgabenwahrnehmung erfolgte. Auch die zweite

${ }^{46}$ Die Grundgesamtheit bildeten 6.590 Maschinen- und Anlagenbaubetriebe ab einer Größe von 21 Beschäftigten auf Basis einer Adressdatei der BA. Postalisch angeschrieben wurden zufällig gezogene 2.171 Betriebe mit einem disproportionalen Schichtungskonzept. Auswertungsgrundlage war letztlich eine Nettostichprobe von 726 Fragebögen mit einer höheren Gewichtung der kleinen Betriebe, was nach erfolgter Bereinigung (z. B. Abzüge der Betriebe ohne Betriebsratsgremium) einer Stichprobenausschöpfung von $38 \%$ entsprach (Müller-Jentsch \& Seitz, 1998, S. 362 ff.).

${ }^{47}$ Die Summe wurde wie folgt gebildet: spezielle Arbeitsinhalte wie Technikgestaltung oder Arbeitsorganisation (4 Punkte), dazu abgeschlossene Vereinbarungen (8 Punkte), vorhandene Gruppenarbeit im Betrieb, deren Beurteilung und Ausgestaltung (6 Punkte), Qualitätszirkel/moderne Arbeitszeitregelungen (2 Punkte) und Verhandlungen über die Arbeitszeit und eine Beschäftigungsgarantie (4 Punkte) (Müller-Jentsch et al., 1998, S. 83). Mit insgesamt neun Punkten liegt in dem Index eine deutliche Gewichtung auf abgeschlossenen Betriebsvereinbarungen. 
Typenbeschreibung bleibt etwas nebulös. ${ }^{48}$ Die Typen des ,ambitionierten Betriebsrats“ und des „Co-Managers“ charakterisieren sich schließlich durch ein stärkeres einflussnehmendes und proaktives Verhalten im Bereich neuer Arbeitsthemen. Nach den Ergebnissen waren diese insgesamt seltener und häufiger in größeren Betrieben zu finden (ebd., S. 85 ff.).

\section{Betriebsratsinterne Organisation und Arbeitsstrukturierung}

Laut Minssen und Riese (2007, S. 19 ff.) thematisieren vorliegende Typologien zur Mitbestimmungspraxis primär die Beziehungen zwischen dem Betriebsrat und der Führungsebene sowie betriebliche Einflussprozesse. Demgegenüber richten sie ihr Forschungsinteresse auf die betriebsratsinterne Arbeitsorganisation, der sie eine hohe Bedeutung zusprechen, um im Sinne einer Aufgabenbewältigung effektiv zu sein und die zunehmend komplexer werdenden Anforderungen an Betriebsräte zu meistern. Den Bezugsrahmen ihrer Arbeit bilden organisationstheoretische Annahmen sowie die Betriebsrätetypologie von Müller-Jentsch et al. (1998). Erweitert ist diese um die Betrachtung der internen Organisationsstrukturen der Gremien.

Ihre Fragestellungen handeln davon, wie Betriebsräte ihre Arbeit organisieren. Dabei gehen sie von der Annahme aus, dass sich Betriebsräte jenseits der formalen Regelungen des BetrVG durch den geschickten Aufbau und die Pflege von Netzwerkbeziehungen Machtressourcen und eine betriebliche Einflussnahme verschaffen können (Minssen \& Riese, 2007, S. 28). Da ihre Argumentationsgrundlagen zudem darauf beruhen, dass sich eine Einflussnahme erst herauskristallisiere, wenn betriebliche Umstrukturierungen anstehen, wählten sie den Öffentlichen Personennahverkehr (ÖPNV) als Forschungsbereich, der vor anhaltenden Modernisierungserfordernissen steht (ebd., S. 35). ${ }^{49}$ Ihre Einteilung der Betriebsratstypen

\footnotetext{
${ }^{48}$ Die Gruppe ,setzt sich aus Betriebsräten zusammen, die sich zumindest mit neuen Themen beschäftigen oder eine Betriebsvereinbarung darüber abgeschlossen oder sich auf neue Formen der Arbeitsorganisation oder ein flexibles Arbeitszeitmodell eingelassen haben, auf die sie Einfluß zu nehmen versuchen“ (Müller-Jentsch et al., 1998, S. 84). Der Indexkonstruktion ist jedoch zu entnehmen, dass 2-5 Punkte auch über betriebsbeschreibende Aspekte erreicht werden können, aus denen noch keine Auskunft über ein einflussnehmendes oder engagiertes Betriebsratsverhalten hervorgeht (u. a. „Gibt es unter der angeschriebenen Adresse Qualitätszirkel?"; „Gibt es in Ihrem Betrieb Arbeitsgruppen?“; bei vorhandenen Arbeitsgruppen: „Die Gruppensprecher werden von den Mitarbeitern der jeweiligen Gruppen gewählt“; ebd., Anhang 1, S. 1 ff.). Inwieweit solche Fälle unter den erfassten 313 „Engagierten Betriebsräten“ auftreten oder mit welchen Auswertungsmethoden die Typenbeschreibung genau erfolgte, wird im Ergebnisbericht nicht transparent.

49 Grundgesamtheit ihrer schriftlichen Befragung waren 473 Betriebsräte in ÖPNV-Betrieben. In ihrer Vollerhebung aus dem Jahr 2003 konnten sie einen Rücklauf von $30 \%$ erzielen. Diesen beschreiben sie im Abgleich ,wichtiger Strukturdaten (etwa Betriebsgröße, Ost-/West-Verteilung)“ (Minssen \& Riese, 2007, S. 45) als repräsentativ. Inhaltlich ergänzt werden ihre Auswertungen um Ergebnisse aus sechs leitfadengestützten Interviews mit Betriebsräten der polarisierenden Typen und von teilnehmenden Beobachtungen
} 
erfolgte mit dem mehrdimensionalen Index von Müller-Jentsch et al. (1998), wobei die Indikatoren auf den ÖPNV-Bereich ausgerichtet waren. In ihrer Ergebnisdarstellung erinnern Minssen und Riese (2007, S. 51) zunächst daran, dass die Bezeichnung „Co-Manager“ keine kooperativ-gefälligen Verhaltensweisen symbolisiert, sondern dass diese anlässlich der hohen Kontaktdichte zwischen Betriebsrat und Management gewählt wurde, die durch ein proaktives, einmischendes Verhalten der Interessenvertretung entsteht. Mit Bezug auf vergleichbare Forschungsergebnisse konstatieren die Forscher einen leichten Anstieg der aktiven und einflussnehmenden Betriebsratstypen im Bereich des ÖPNV. So liegen die Anteile der zwei stärkeren Betriebsratstypen in den Ergebnissen von Minssen und Riese (2007, S. 49 f.) bei $26 \%$ (ambitionierter Betriebsrat) und $14 \%$ (Co-Manager) im Vergleich zu 19\% und $7 \%$ bei Müller-Jentsch et al. (1998, S. 85). In der Analyse zeigt sich eine deutliche Korrelation dieser Typen zu großen Unternehmen. Lediglich in Betrieben mit weniger als 700 Mitarbeitenden tritt der konventionelle Betriebsrat in Erscheinung. Um einflussnehmende Drittvariablen zu vermeiden, schlossen die Forscher deswegen alle Unternehmen ab einer Größe von $>700 \mathrm{Be}-$ schäftigten (15\% der Betriebe) von ihrer Auswertung aus (Minssen \& Riese, 2007, S. 54).

Kennzeichnend für die Betriebsräte des Typs „Co-Manager“ sind die oftmals tägliche oder wöchentliche Kontaktfrequenz mit der Geschäftsführung, die auch als Strategie verwendet wird, um eigene fachliche Positionen in das Betriebsgeschehen einzubringen (ebd., S. 96 f.). Nach der Ergebnisanalyse liegen für die Co-Manager deutliche Unterschiede in ihrer Arbeitsorganisation vor. In der Arbeitssteuerung haben sie häufiger eine alltägliche Arbeitsplanung und Arbeitsteilung, die auch formalisiert in der Geschäftsordnung zum Ausdruck kommt. Sie strukturieren ihre Arbeit mit Ausschüssen, die eine große Themenbreite aufweisen, und steuern die eigene Arbeit häufiger über Zielvereinbarungen und Feedbackrunden (ebd., S. 66 f., S. 129). Beständiger und umfangreicher fällt auch die betriebsinterne Kommunikation zu den Betriebsangehörigen aus. So gelingt es diesem Betriebsratstyp, die Belegschaft in seine Arbeit zu integrieren und damit zugleich seine personellen Ressourcen zu erhöhen, die im Umfang nicht von den mittleren Typen (engagierter und ambitionierter Betriebsrat) abweichen. Keine Unterschiede liegen bei der Gestaltung der Betriebsratssitzzungen (etwa Einhaltung der formalisierten Abläufe, wechselnde Moderation), der externen Einbindung und Netzwerke (es

(Betriebsrätesitzungen) (ebd., S. 47). Operationalisiert wurden ihre Fragestellungen u. a. über Indikatoren zur Gründung und Beteiligung in Arbeitssauschüssen, zu Inhalten der Geschäftsordnungen, der Kommunikation zur Belegschaft und Geschäftsführung, wobei sie mit auffällig vielen Einschätzungs- und Bewertungsskalen arbeiteten. 
zeigt sich lediglich ein ausdifferenzierterer Kontakt des einflussreichsten Betriebsratstypus mit Betriebsräten anderer Unternehmen) oder dem Wahlerfolg vor (ebd., S. 81, S. 112 ff.). Sehr auffallend ist der Weiterbildungsumfang. In einer Amtszeit besuchten die Mitglieder dieses Typs doppelt so viele Angebote (ca. 11 Veranstaltungen) wie diejenigen der anderen Typen. Die gremiumsinterne Wissensvermittlung wird zudem durch eine größere Bandbreite von Maßnahmen (z. B. Einzelschulungen, Referate) abgesichert (ebd., S. 103 ff.).

\section{Abgeschlossene Betriebsvereinbarungen}

Bereits in der frühen Mitbestimmungsforschung findet sich die Hypothese, dass ein Betriebsrat nur ,als interessenwirksam im Sinne der Arbeitnehmer“ (Diefenbacher \& Nutzinger, 1980, S. 103) gelten kann, wenn er Betriebsvereinbarungen abgeschlossen hat. Zwar ist zu berücksichtigen, dass eine Abschlussverweigerung ein Machtmittel des Betriebsrats bilden kann und eine hohe Anzahl von Vereinbarungen ggf. Ausdruck einer unternehmerischen Managementstrategie ist (ebd.), dennoch gelten Betriebsvereinbarungen als Indikator für betrieblichen Einfluss und Konsensfindung (Müller-Jentsch et al., 1998, S. 64). Von Nienhüser (2005) liegt eine Arbeit vor, die mit Betriebsvereinbarungen verbundene Prozesse erforscht. In dieser wird die Anzahl vorliegender Vereinbarungen im Hinblick auf die wahrgenommene Macht und Kooperationsbereitschaft des Betriebsrats aus Sicht des Betriebsmanagements analysiert und untersucht, wie sich Bewertungen von Betriebsvereinbarungen dazu verhalten. Ausgangshypothese bildete die Annahme, dass die Art und Weise, wie ein Betriebsrat wahrgenommen wird, zu unterschiedlichen Abschlusszahlen betrieblicher Vereinbarungen führt (ebd., S. 6 ff.). Analysebasis waren 1.000 Telefoninterviews mit Personalverantwortlichen von Betrieben mit einer Größe von mehr als 100 Beschäftigten. ${ }^{50}$ Anhand der Dimensionen Macht und Kooperationsbereitschaft bildete Nienhüser vier Typen von Betriebsräten. ${ }^{51}$ Beide

\footnotetext{
${ }^{50}$ Anvisiert waren Befragungspersonen, denen der Abschluss von Betriebsvereinbarungen oblag, oder Personen der höchstmöglichen Managementebene. Ziehungsgrundlage der geschichteten Stichprobe war die Hoppenstedt-Firmeninformationsdatei. Ausgeschlossen wurden Betriebe ohne Betriebs-/Personalrat sowie drei spezifische Wirtschaftszweige (sonstige öffentliche und persönliche Dienstleistungen, Landwirtschaft und verwandte Zweige, Erziehung und Unterricht; Nienhüser, 2005, S. 6 ff.).

${ }^{51}$ Die Operationalisierung von „Macht“ beruhte auf drei Einschätzungsfragen. Bei diesen wurde jeweils ein Punkt bei hohen Bewertungen vergeben. Der sich insgesamt ergebende Wertebereich von 0 bis 3 wurde anschlieBend in die dichotome Gliederung, ,hohe“ und ,geringe Macht“ überführt. Ein ähnliches Vorgehen wurde für das Konstrukt „Kooperationsbereitschaft“ gewählt, wobei drei Statements aus bereits angewandten Erhebungsinstrumenten angewandt wurden (Nienhüser, 2005, S. 15 f.).
} 
Dimensionen konnten entweder die Ausprägung hoch oder gering einnehmen, womit sich die in Tabelle 6 dargestellten Kombinationsmöglichkeiten ergaben.

Tabelle 6: Zweidimensionale Betriebsratstypologie von Nienhüser (2005)

\begin{tabular}{lll}
\hline Betriebsratstyp & $\begin{array}{l}\text { In Prozent } \\
(n=959)\end{array}$ & $\begin{array}{l}\text { Anzahl Betriebsverein- } \\
\text { barungen }(n=945)\end{array}$ \\
\hline I: Stark, kooperativ & $19 \%$ & 17.3 \\
II: Stark, wenig kooperativ & $20 \%$ & 19.4 \\
III: Schwach, kooperativ & $37 \%$ & 11.9 \\
IV: Schwach, wenig kooperativ & $22 \%$ & 10.5 \\
\hline Gesamt & $100 \%$ & 14.1 \\
\hline
\end{tabular}

Anmerkung. Eigene Anfertigung nach Nienhüser (2005, S. 18 f.).

Nach dieser Klassifizierung sind die starken Betriebsratstypen sowie der schwache und zugleich wenig kooperative Betriebsrat in etwa gleichem Maße verbreitet, hingen ist Typ III (schwach und kooperativ) besonders häufig anzutreffen. In fast allen befragten Firmen lagen abgeschlossene Betriebsvereinbarungen vor $(99 \%$, arithmetischer Mittelwert $[M]=14$, Standardabweichung $[S D]=19$ ). Im Mittel sind diese besonders häufig bei starken Betriebsräten mit geringer Kooperationsbereitschaft anzutreffen, und zwar sowohl bei kleineren als auch größeren Betrieben, was durch die eingeführte Kontrollvariable der Betriebsgröße ersichtlich wurde (ebd., S. 11 ff.). Die Bewertungen von Betriebsvereinbarungen (gemessen an drei Einschätzungsfragen) durch das Management fallen in der Analyse überraschend positiv aus, wobei es wiederum Abweichungen bei den starken, wenig kooperativen Betriebsräten gibt und das Regulierungsinstrument hier schlechter bewertet wird (ebd., S. 18 ff).

Weitergeführt wurde die Ergebnisauswertung in Form von Regressionsanalysen, um mögliche Interaktionseffekte aufzudecken. Gerechnet wurden verschiedene Modelle. Hieraus ergibt sich u. a., dass bei der Anzahl der abgeschlossenen Betriebsvereinbarungen die Stärke eines Betriebsrats eine größere Rolle spielt als dessen Kooperationsbereitschaft. Dieser Effekt der Gremiumsstärke fällt jedoch weit geringer aus als derjenige, der von der Betriebsgröße und dem gewerkschaftlichen Organisationsgrad im Betrieb ausgeht (ebd., S. 21 ff.).

Wie die dargestellten Studien zeigen, liegen zur Arbeit von Betriebsräten Studien vor, die betriebsrätliches Handeln umfänglich untersuchen oder mit sehr spezifischen 
Untersuchungsfragen aufgreifen. Grundlegend anders ist demgegenüber der Forschungsstand zur Werkstattratsarbeit.

\subsection{Ergebnisse zur Beteiligung in WfbM}

Um den marginalen Wissensstand zur Beteiligung der Beschäftigten mit Behinderungen in WfbM vorzustellen, wird in diesem Abschnitt ein Bogen gespannt von der Mitwirkung mit offenen Vorgaben in den 1990er-Jahren (Abschnitt 4.2.1) über erste Erfahrungen nach Einführung der WMVO im Jahr 2001 (Abschnitt 4.2.2) bis zu ergänzenden Hinweisen aus Praxisprojekten (Abschnitt 4.2.3).

\subsubsection{Mitwirkung auf Basis offener Vorgaben}

Wie in Abschnitt 3.3.1 dargestellt, wurde die fachliche Anforderung zur Mitwirkung der Beschäftigten mit Behinderungen in den Werkstätten zunächst nach eigenem Gutdünken umgesetzt. Zur Sondierung der Umsetzung gab das Bundesministerium für Arbeit und Sozialordnung Ende der 1980er-Jahre eine Studie in Auftrag. In dieser wurden eine schriftliche Fragebogenerhebung und qualitative Leitfadeninterviews mit verschiedenen Interessengruppen in 15 WfB realisiert. Die quantitative Befragung ist nach Meinung der durchführenden Mitbestimmungsforscher Breit und Kotthoff (1990) jedoch verengt und von den Trägerverbänden beeinflusst gewesen. So hätten Fragen zu dem sozialen Kontext, der Unternehmenskultur sowie zu werkstattpolitischen Themen entfallen müssen (ebd., S. 9). In der Durchführung wurden auf Basis einer Adressliste alle damaligen Werkstätten angeschrieben (mit Ausnahme der 15 Werkstätten, die an der qualitativen Teilstudie teilgenommen hatten), insgesamt 372 Einrichtungen. Diese erhielten einen Fragebogen für die Leitung und einen separaten Bogen für das Gremium der Interessenvertretung. Bezogen auf die Werkstättenzahl lag der Rücklauf bei 57 \% (212 Werkstätten), mit deutlichen Unterschieden nach Trägerverbänden (LebenshilfeWerkstätten: $45 \%$; Caritas-Werkstätten: $78 \%$ ).

Von den antwortenden Werkstätten hatten mehr als $80 \%$ ein an demokratischen Prinzipien orientiertes Mitwirkungsgremium der Menschen mit Behinderungen in WfB eingerichtet (im Weiteren als Werkstattrat bezeichnet). In den Werkstätten, in denen dies nicht der Fall war, wurde die Nichteinrichtung u. a. damit begründet, dass dies behinderungsbedingt nicht möglich sei und eine Pro-forma-Vertretung als Manipulation begriffen werde (ebd., S. 39 f.). 
Zur Beschreibung der vorhandenen Werkstatträte lagen 168 Fragebögen vor. ${ }^{52}$ Zwei Drittel der Werkstatträte führten mindestens einmal im Monat Sitzungen durch. $78 \%$ verfügten über eine schriftliche Arbeitssatzung (ebd., S. 14 f.). Trotz des hohen formalen Institutionalisierungsanteils war eine Mitwirkung der Gremien vonseiten der Leitungen kaum beabsichtigt. Mitwirkung wurde ,von etwa der Hälfte der Einrichtungsleitungen noch keineswegs ernsthaft akzeptiert“ (ebd., S. 152). Werkstättenintern nahmen Werkstatträte zumeist eine geringe Bedeutung ein. Die vorhandenen Aktivitäten bezogen sich primär auf lebensweltliche Themen (bspw. Freizeitgestaltung, Einrichtungsordnung) und weniger auf betriebliche Aufgabenbereiche (bspw. Arbeitsabläufe oder das Arbeitstraining). Die Reichweite der Beteiligung blieb auf eine bloße Informierung und Anhörung beschränkt, nur wenige Werkstatträte brachten Beschwerden und Vorschläge an die Werkstattleitung ein, wobei noch keine Verfahrensabläufe festgelegt waren, wie mit dieser Form der Einbringung umzugehen sei (ebd., S. 152 ff.).

Im Gesamtblick der quantitativen Erhebungsergebnisse charakterisierten Breit und Kotthoff drei unterschiedliche Mitwirkungsprofile. Aus den qualitativen Daten leiteten sie wiederum vier verschiedene Typen von Werkstatträten ab, die sie den Profilen zuordneten. Anhand einer fallweisen, materialorientierten individuellen Zuordnung jedes einzelnen Gremiums ergab sich die in Tabelle 7 angegebene Verteilung.

Nach den Ergebnissen waren lediglich $15 \%$ der Werkstatträte aktiv und bedeutungsrelevant. Fast die Hälfte wurde hingegen als bedeutungslose Interessenvertretung klassifiziert. Besonders prekär erscheint der Befund, dass die Mitglieder dieser Werkstatträte zu disziplinarischen Maßregelungen gegenüber ihrer Vertretungsbasis neigten und eine Rollenwahrnehmung als „Hilfs-Sheriffs“ (ebd., S. 41) verbreitet war. Die klassifizierte Ausprägung der Mitwirkung sei wiederum kontextspezifisch in der „Arbeits- und Sozialstruktur der WfB“ (ebd., S. 129) zu sehen und erklärbar, wie Breit und Kotthoff ihre Ergebnisse analysieren. Werkstatträte in stark produktionsorientierten WfB hätten die schwierigsten Ausgangsbedingungen. Werkstatträte in rehabilitativ-pädagogisch ausgerichteten Werkstätten fänden demgegenüber eher die Unterstützung und Förderung durch die Leitung und das Stammpersonal. Hierbei sei wiederum eine Normalitäts- gegenüber einer starken Betreuungsorientierung vorteilhaft. Dies wurde als prägnanter Unterschied zur betrieblichen Mitbestimmung in Betrieben des allgemeinen Arbeitsmarkts festgehalten: „Der tragende Boden der

\footnotetext{
52 Nicht berücksichtigt wurden Bögen ohne Angaben der Leitung, zusätzliche 22 Bögen für Interessenvertretungen in separaten Betriebsstätten wurden in Teilauswertungen einbezogen (Breit \& Kotthoff, 1990, S. 10).
} 
Behindertenmitwirkung ist die sozialpädagogische Einstellung der Leitung und des Stammpersonals, nicht der Interessenkampf“" (ebd., S. 151).

Tabelle 7: Werkstattratstypen und Mitwirkungsprofile von Breit und Kotthoff (1990)

\begin{tabular}{|c|c|c|}
\hline $\begin{array}{l}\text { Mitwirkungsprofil } \\
\text { Werkstattratstyp }\end{array}$ & // Beschreibung & $\begin{array}{l}\text { In Prozent } \\
(n=168)\end{array}$ \\
\hline $\begin{array}{l}\text { Profil I } \\
\text { Typ A }\end{array}$ & $\begin{array}{l}\text { - Aktivitäten beziehen sich sowohl auf lebensweltliche } \\
\text { - Hohs auch beschäftigungsrelevante Bereiche. } \\
\text { kungsorganisation und -prozesse. } \\
\text { - Bringen Vorschläge und Beschwerden ein. } \\
\text { - Unterstützung durch die Einrichtungsleitung und das } \\
\text { - Stammpersonal ist vorhanden. } \\
\text { - Nehmen Einfluss auf die „Innenpolitik“ der WfB. }\end{array}$ & $15 \%$ \\
\hline $\begin{array}{l}\text { Profil II } \\
\text { Typ B }\end{array}$ & $\begin{array}{l}\text { - Sind in wenigen Bereichen aktiv. } \\
\text { - Geringere Selbstständigkeit bei der Umsetzung der } \\
\text { - Kitwirkungsorganisation und der -prozesse. } \\
\text { - Keine kontinuierliche Arbeit. } \\
\text { - Geringe soziale Bedeutung in der WfB. }\end{array}$ & $38 \%$ \\
\hline $\begin{array}{l}\text { Profil III } \\
\text { Typ C und D }\end{array}$ & $\begin{array}{l}\text { - Sehr geringe Bedeutung als Vertretung der Menschen } \\
\text { mit Behinderungen in WfB. } \\
\text { - Fehlende Selbstständigkeit bei der Umsetzung der Mit- } \\
\text { wirkungsorganisation und der -prozesse. } \\
\text { - Vertrauensperson fungiert als Stellvertretung. } \\
\text { - Werden nicht beachtet oder als „Disziplinierungshel- } \\
\text { fer*in“ eingesetzt. }\end{array}$ & $47 \%$ \\
\hline
\end{tabular}

Anmerkungen. Eigene Anfertigung nach Breit \& Kotthoff (1990, S. 149 ff.); zur Generierung einer hohen Erhebungsbeteiligung der eigenen Studien in ähnlicher Art bereits veröffentlicht (Schachler, 2019, S. 48).

Neben dem Klima in den Werkstätten und den Beziehungen zwischen Werkstattrat und dem Werkstattpersonal wurden weitere Komponenten benannt, die das Ausmaß der Beteiligung beeinflussen: die Verteilung der Behinderungsarten im Werkstattrat, überdurchschnittliches Engagement von Einzelmitgliedern, das Rollenverständnis der Vertrauensperson, eine tragfähige Beziehung zur Belegschaft mit Behinderungen, Schulungen für Werkstatträte (ebd., S. 15 ff.). Zu beachten ist, dass diese Analyseergebnisse einem Zeitpunkt entstammen, zu dem 
das Mitwirkungsgeschehen in Werkstätten noch nicht geregelt war, und sich der formal-rechtliche Rahmen seitdem grundlegend verändert hat.

\subsubsection{Regionale Studien im Anschluss an die Einführung der WMVO}

\section{Regionale Studie im Raum NRW (Schlummer, 2004)}

Von Schlummer (2004) liegt eine regionale Pilotstudie zwei Jahre nach Inkrafttreten der WMVO zu den Erfahrungen und zur Zusammenarbeit von Werkstattrat, Werkstattleitung und Vertrauenspersonen vor. Die als Situationsbeschreibung angelegte Fragebogenerhebung richtete sich an 83 der damaligen $135 \mathrm{WfbM}$ in NRW. Angeschrieben wurden alle Einrichtungen, aus deren Namen nicht offensichtlich eine kirchliche Trägerschaft hervorging. Es sollten lediglich Werkstätten ohne konfessionelle Bindung befragt werden, da sich die Verordnungen der Caritas und der Diakonie noch im Umsetzungsprozess befanden. Dieser angedachte Ausschluss ließ sich jedoch nicht realisieren, ca. ein Drittel des Erhebungsrücklaufs stammte aus kirchlichen Einrichtungen. Verwendet wurden drei unterschiedliche Fragebögen pro Werkstatt: einer für die Werkstattleitung, einer für den Werkstattrat und einer für die Vertrauensperson. Im Ergebnis lagen für die Teilstudien zwischen 41 und 45 Bögen vor; der Rücklauf betrug $49 \%$ (Werkstattleitungen), $53 \%$ (Vertrauenspersonen) und $54 \%$ (Werkstatträte) (ebd., S. 59 ff.).

Die Daten der Untersuchung liefern Informationen $\mathrm{zu}$ den Rahmenbedingungen der WMVO und Einschätzungen von den bei den Mitwirkungsprozessen beteiligten Personengruppen. Es zeigt sich z. B., dass in ca. 70 \% der WfbM mindestens monatliche Austauschgespräche zwischen Werkstattrat und Werkstattleitungen stattfinden und ebenfalls ca. $70 \%$ der Werkstatträte ihre Interessen durch die Leitung als ausreichend berücksichtigt sehen. Demgegenüber bewerteten nur 50 \% der Vertrauenspersonen die Zusammenarbeit zwischen den beiden als gut oder sehr gut (ebd., S. 69). Beachtenswert ist weiter, dass zum damaligen Zeitpunkt lediglich $15 \%$ der befragten WfbM über eine eingerichtete Vermittlungsstelle verfügten (4 von $26 \mathrm{WfbM}$, ohne Einrichtungen der Diakonie und Caritas). In der Ergebnisinterpretation greift Schlummer die (pädagogische) Argumentation auf, dass die Arbeit als Werkstattrat (hoch-)komplexe Aufgaben beinhaltet, für die entsprechende, umfangreiche (Bildungs-)Angebote zum Kompetenzerwerb notwendig sind (ebd., S. 78 ff.). 
Regionale Studie in Bayern (Hoffmann, 2012)

An die Bestandsaufnahme zur Umsetzung der WMVO in NRW schließt sich die Forschungsarbeit von Hoffmann (2012) an. Dieser untersuchte 2010 mit den Instrumenten von Schlummer (2004) den Stand der Mitwirkungspraxis in bayerischen WfbM. Angeschrieben wurden alle zum Erhebungsstand bestehenden anerkannten WfbM in diesem Bundesland $(N=102)$. Erzielt wurde eine Ausschöpfungsquote von 34 \% (Vertrauenspersonen und Werkstattleitungen) bzw. $42 \%$ (Werkstattrat). Im Vergleich zur Studie von Schlummer fanden zum Erhebungszeitpunkt bei den bayerischen Werkstatträten die mindestens monatlichen Gespräche mit der Leitung etwas seltener statt. Bei $35 \%$ waren diese nicht gegeben (bei Schlummer 30 \%). Es bestätigten sich die Befunde, dass Werkstatträte kaum Kontakte zu Gewerkschaften pflegen (Schlummer: $7 \%$ ), Kontakt zur werkstattinternen Arbeitnehmervertretung (Betriebsrat/MAV/Personalrat) hingegen häufiger gegeben ist (Schlummer: $50 \%$; Hoffmann: $60 \%$ nach Angaben der Vertrauenspersonen). Prekär erscheint das Ergebnis, dass rund die Hälfte der Vertrauenspersonen noch an keiner Schulung für ihre anspruchsvolle Tätigkeit teilgenommen hatte (Hoffmann, 2012, S. 16 ff.).

\section{Feldepisoden zur Werkstattratsarbeit (Lahoda, 2018)}

Die in Abschnitt 3.1.3 dargestellte kulturwissenschaftliche Feldforschung zur Arbeit in WfbM beinhaltet dezidierte Beschreibungen eines Werkstattrats aus den Jahren 2011 und 2012. Dieses Gremium wurde 1999 etabliert und bestand zum Erhebungszeitpunkt aus fünf Personen. Ein Mitglied war seit der Etablierung mit dabei. Die Vertrauensperson gehörte dem Fachdienst an (Lahoda, 2018, S. 293 ff.). In der Mikrostudie sind Werkstattversammlungen sowie deren Vorbereitungen ausführlich dargestellt. Bereits die Beschreibungen der Vorbereitungen präsentieren eine direktive und unreflektierte Beziehung zwischen der Vertrauensperson und dem Werkstattrat. Bei der Werkstattversammlung sind ca. 170 Beschäftigte anwesend. An dieser kann während der Arbeitszeit freiwillig teilgenommen werden. Als Teilnahmemotivation werden die Informationsmöglichkeiten und eine Alltagsfluktuation (,Alles besser als Arbeiten“; ebd., S. 296) angeführt. Inhaltlich werden auf der Versammlung Informationen weitergegeben, Jubilare geehrt, Fragen angesprochen und teilweise beantwortet. Zunächst kommen die Vertrauensperson und der Werkstattrat zu Wort. Anschließend wird über die wirtschaftliche Lage und anstehende Veränderungen durch einen Gruppenleiter und die Werkstattleitung berichtet. Als eine Werkstatträtin eine Beschwerde anspricht, in der es um das Verhalten des Fachpersonals geht (Rauchen während der Arbeitszeit, was für Werkstattbeschäftigte verboten 
ist), verweigert der Werkstattleiter eine Stellungnahme. Zwischen den Berichten sind Fragen durch die Werkstattbeschäftigten zulässig. Diese zeigen ein umfangreiches Interesse an Arbeitsthemen (ebd.).

In der Versammlungsdurchführung setzt sich die direktive Beziehung zwischen der Vertrauensperson und dem Werkstattrat fort, wie u. a. die folgenden Ausschnitte offerieren

„Wie zuvor geprobt, beginnt die Werkstattversammlung nach der Einführung durch die Vertrauensperson .... Die Fragen werden zum Großteil von der Vertrauensperson oder vom Werkstattleiter Nicolai Gebel kurz beantwortet. ... der Werkstattrat als auch die Beschäftigten im Publikum stehen selbst kaum in direktem Austausch ohne Einfluss des Werkstattpersonals.“ (ebd., S. 296)

Lahoda schlussfolgert daraus, dass für die Veranstaltungsdurchführung eine elementare Unterstützung des Werkstattrats notwendig sei (ebd., S. 299). Dass die vorhandenen Unterstützungsleistungen kaum auf eine zu erzielende Eigenständigkeit ausgerichtet sind und direktive, fast übergriffig anmutende Verhaltensweisen offenbaren, reflektiert sie nicht. Beispielsweise hat bereits die Versammlungseröffnung durch das Fachpersonal und nicht den Werkstattrat symbolischen Charakter, ein Umgang mit Fragen könnte selbstreflexiv erarbeitet werden usw.

Aus den Beschreibungen der Ausstattungsbedingungen des Werkstattrats geht hervor, dass dieser räumlich und strukturell in der Werkstatt schlecht verortet ist. So hat der Werkstattrat eine E-Mail-Adresse, aber keinen Raum (mit Computer), den er nutzen kann. Ein gut zugänglicher Schrank wurde erst kürzlich eingerichtet. Eine Freistellung der Vorsitzenden besteht nicht (ebd., S. 304). Seit Kurzem verfügt der Werkstattrat über ein eigenes Budget für seine Arbeit, das er erfolgreich beantragt hat. Er möchte dies jedoch nicht selbst verwalten. Dies sei

„noch ein zu großer Schritt ... dem sich der Werkstattrat nicht gewachsen fühlt. Glücklicherweise zeigt sich der Werkstattleiter ... verständnisvoll, er könnte genauso darauf beharren, ihnen ab jetzt die gesamte Verantwortung für ihr Budget zu übertragen." (ebd., S. 302)

Nicht erwähnt werden eine genauere Ursachenforschung oder Reflexion darüber, welche Bedenken des Werkstattrats zur Budgetverwaltung vorliegen, inwieweit die Vertrauensperson dazu ermutigen kann, ob es mögliche Zwischenschritte zur Verantwortungsübernahme gibt etc. Auffällig ist, dass in den Formulierungen lediglich von einer Mitwirkung des Werkstattrats geschrieben wird, obwohl zum Erhebungszeitpunkt nach der DWMV bereits Mitbestimmungsrechte vorgesehen waren. Die Instanz der Vermittlungsstelle und deren Funktion wird nicht erwähnt. Insgesamt zeichnet Lahoda ein Bild der Werkstattratsarbeit, das von erheblichen Defiziten in der Einbindung und der Akzeptanz des Gremiums als Akteur des Werkstattgeschehens zeugt. 


\subsubsection{Hinweise aus Praxisprojekten}

Erste Erfahrungen (Bieneck \& Engelmeyer, 2004)

Aus einem Projekt zur Erstellung einer Fortbildungskonzeption für Werkstatträte und von Unterlagen, die als Wahlhilfen verwendet werden können, liegen umfängliche Beobachtungen zur Werkstattratsarbeit aus den Jahren 2002 und 2003 vor (Bieneck \& Engelmeyer, 2004). Festgehalten wurde u. a.:

- $\quad$ eine noch bestehende Zurückhaltung bei der inhaltlichen Befassung mit der WMVO (insbesondere bei Leitungen, aber auch bei Werkstatträten) und große Unterschiede im Kenntnisstand zur WMVO;

- eine Defizitorientierung des Fachpersonals bezogen auf das Vermögen von Werkstatträten, ihr Amt der Interessenvertretung auszufüllen. Beschrieben sind zwei Phänomene: zum einen die Beobachtung, Werkstatträten ihre vorgesehenen Aufgaben wohlmeinend oder paternalistisch abzunehmen, zum anderen das Phänomen, den Mitgliedern des Werkstattrats eine Rolle als „Seelsorger“ o. Ä. für die Werkstattbeschäftigten zuzuweisen (ebd., S. 26);

- $\quad$ eine noch unklare Finanzierungspraxis der Sachkosten des Werkstattrats (insbesondere zu den Freistellungen der vorsitzenden Person und der Vertrauensperson);

- Probleme mit der als gering empfundenen Mitgliederanzahl und dem Umstand, dass laut WMVO Werkstatträte nicht in Zweigwerkstatträten gewählt werden. Dies führe zu einer Arbeitsüberforderung von Mitgliedern und die Vertretung der Interessen von Zweigwerkstätten sei nicht mehr sichergestellt;

- potenzielles Konfliktpotenzial und Schwierigkeiten bei der Rollenfindung von Vertrauenspersonen aus dem Fachpersonal;

- ein großer Fortbildungsbedarf zur Umsetzung der WMVO bei Werkstatträten, Leitungen, Fachpersonal und Vertrauenspersonen (ebd., S. 25 ff.).

\section{Umfrage von Werkstatträte Deutschland (BVWR, 2013)}

Ergänzend zu den Beobachtungen aus der Anfangspraxis mit der WMVO lässt sich auf eine Befragung der bundesweiten Interessenvertretung Werkstatträte Deutschland e. V. aus den Jahren 2012 und 2013 verweisen (zum damaligen Zeitpunkt noch BVWR e. V. genannt). Inhalte waren die Arbeitsausstattung und die Einbindung der Gremien. Verschickt wurden 1.070 Fragebögen an Werkstatträte. Die Grundgesamtheit bleibt etwas unklar. Vermutlich wurden 
die Fragebögen an alle Mitglieder der LAGen der Werkstatträte geschickt, mit Ausnahme von Thüringen und Sachsen, in denen zum damaligen Zeitpunkt keine Landesvertretungen aktiv waren. Erzielt wurde ein Rücklauf von 40 \% (425 Fragebögen).

Die Befragungsergebnisse verdeutlichen, dass mehr als zehn Jahre nach der Verabschiedung der WMVO eine gute Ausstattung und Einbindung des Gremiums noch keine Selbstverständlichkeiten waren: Über $70 \%$ der Befragten hatten kein eigenes Büro für den Werkstattrat. Rund 35 \% verfügten nicht einmal über einen abschließbaren Schrank für die vertraulichen Dokumente. Beachtliche $21 \%$ der Werkstatträte führten nicht einmal Werkstattversammlungen durch etc. (BVWR, 2013, S. 3 ff.).

\section{Aktuelle Arbeitsmaterialien und Ratgeber für Werkstatträte}

Zur Erklärung der neuen Regelungen der WMVO ab 2017 (siehe Abschnitt 3.3.2) liegen verschiedene Arbeitsmaterialien für Werkstatträte vor. Keine Materialien sind der Verfasserin hingegen für Werkstattleitungen bekannt. Gleichfalls ist die Lage der aktuelleren Handreichungen für Vertrauenspersonen überschaubar. ${ }^{53}$

Als Projektergebnis neueren Datums besteht ein „Ratgeber für Werkstatt-Räte von Werkstatt-Räten“ (Diakonisches Werk Rheinland-Westfalen-Lippe e. V. - Diakonie RWL \& Deutscher Paritätischer Wohlfahrtsverband Landesverband Nordrhein-Westfalen e. V., 2019). Dieser erklärt die Arbeit mit der WMVO in Leichter Sprache, greift Themen der praktischen Arbeit auf (z. B. Kapitel 6 „Wie bearbeitet der Werkstatt-Rat eine neue Anfrage?“) und enthält dazu passende Vorlagen. Entstanden ist der Ratgeber im Rahmen des dreijährigen Projekts „Partizipation und Selbstvertretung von Menschen mit Behinderung im arbeitnehmerähnlichen Rechtsverhältnis“ im Auftrag der freien Wohlfahrtspflege und weiterer Beteiligter (Laufzeit 2016 bis 2019). In der Durchführung nahmen fünf Werkstätten teil, die selbstgewählte Themen bearbeiteten und dazu passende Arbeitsmittel erstellten, mit denen der Werkstattrat seine (gewachsenen) Aufgaben bewältigen kann. Begleitet wurde das Projekt durch eine Evaluation der „Gesellschaft für Forschung und Beratung im Gesundheits- und Sozialbereich mbH“, die u. a. leitfadengestützte Einzel- und Gruppengespräche in den projektteilnehmenden Werkstätten durchführte. Die Stärke dieser Evaluation kann in der „handlungsorientierten

${ }^{53} \mathrm{Zu}$ den Materialien für den Werkstattrat siehe etwa: Werkstatträte Deutschland e. V., verfügbar unter: https://www.werkstatträte-deutschland.de/downloads; Diakonie Deutschland, verfügbar unter: https://www.diakonie.de/in-leichter-sprache-diakonie-werkstaetten-mitwirkungs-verordnung. Zur Arbeit von Vertrauenspersonen BAG WfbM, verfügbar unter: https://www.bagwfbm.de/article/517 
Begleitung“ (Cannizzaro et al., 2020, S. 65) vermutet werden. Die Evaluation ist nicht veröffentlicht. Hinweise kann lediglich ein Zeitschriftenbeitrag geben, in dem Faktoren zusammengefasst werden, die sich positiv auf die Arbeit des Werkstattrats auswirken. Im Kern wird auf die sachliche Ausstattung des Werkstattrats, einen guten Wissensstand zur WMVO und zu weiteren Anlaufstellen verwiesen (ebd., S. 67). Überwiegend werden damit Aspekte aufgeführt (regelmäßige Treffen mit der Leitung, rechtzeitige, umfassende und angemessene Informationen etc.), die in der WMVO vorgesehen und enthalten sind.

Die vorgestellten praxisbezogenen Berichte legen nahe, dass die Vorgaben der WMVO nicht selbstredend umgesetzt werden und nicht alle Werkstatträte ihre gegebenen Rechte nutzen. Um diese Ambivalenz zwischen Normzustand und praktischer Handhabung zu vertiefen, lohnt sich ein kleiner Exkurs in die Rechtssoziologie.

\subsection{Exkurs: Rechtsmobilisierung - Erkenntnisse aus der Rechtssoziologie}

Die Rechtssoziologie richtet den Blick auf die philosophische und reflexive Beschäftigung mit Recht; es geht um die (gesellschaftlichen) Funktionen, die konkrete Umsetzung, die Folgen und die Wirkungen von Recht. Nicht schematisch-juristische Lösungswege, sondern theoretische Erklärungsversuche und empirische Verstehensprozesse stehen im Zentrum des Wissenschaftsinteresses (Baer, 2017, S. 14 ff.). Die Rechtssoziologie geht zurück auf die soziologischen Klassiker; es liegen rechtssoziologische Betrachtungen von Weber, Luhmann und Habermas vor (Fuchs, 2019b, S. 33 ff.). Trotzdem wird die Rechtssoziologie, im weiteren Sinne als interdisziplinäre Rechtsforschung bezeichnet, hierzulande eher marginal betrieben (Rosenstock, Singelnstein \& Boulanger, 2019, S. 4). Als „Arbeit an den Grenzen der Disziplinen“ (Baer, 2016, S. 218) ist die Forschungsrichtung laut den Selbstdarstellungen mit einer Etablierungsmisere und anderen Herausforderungen konfrontiert (ebd.).

Unter der Fülle an Themen wird in der Rechtssoziologie unter der Bezeichnung der Rechtsmobilisierung die tatsächliche, aktive Inanspruchnahme und praktische Anwendung von Recht durch Einzelpersonen oder Gruppen angesprochen (Fuchs, 2019a, S. 245). Der Subjektbezug steht im Vordergrund und die Frage danach, wann und wie es tatsächlich zur Rechtsnutzung kommt, wobei die individuelle oder kollektive Rechtsmobilisierung nicht mit einer Rechtsdurchsetzung gleichgesetzt werden kann. Nicht nur formale (juristische) Verfahrenswege (vor Gericht), sondern auch das persönliche Auftreten und das argumentative Gebaren werden als diskursive Rechtsmobilisierung darunter gefasst (Baer, 2017, S. 226 f.; Fuchs, 2019a, S. 245). Im Prozess der Rechtsmobilisierung wirken verschiedene Komponenten. 
Felstiner, Abel und Sarat (1980) bringen dies mit ihrem Beitragstitel „Naming, Blaming, Claiming“ prägnant zum Ausdruck: Ein Konflikt muss zunächst bemerkt, benannt und als rechtliches Problem externalisiert, d. h. anderen zugeschrieben werden (Fuchs, 2019a, S. 246). Erst dann kann Recht überhaupt geltend gemacht werden. Gerade bei neuen Rechten, die „den bisherigen Routinen und Stereotypen in den meisten Institutionen“ (Kocher, 2013, S. 74) widersprechen, sind häufig erst rechtliche Verfahren notwendig, bis es zur Rechtsdurchsetzung kommen kann (ebd.).

Konsens besteht dahingehend, dass Rechtsmobilisierung durch subjektive und objektive Faktoren beeinflusst wird. Unter den subjektiven Faktoren treten besonders die individuellen Wissensbestände hervor, differenziert als grundlegendes Rechtsbewusstsein, spezifischere Rechtskenntnis und individuelles Anspruchswissen (Baer, 2017, S. 219). Wenngleich die Grenzen zwischen diesen drei Wissenskomponenten etwas fließend bleiben, thematisieren sie drei verschieden gelagerte Bereiche:

- Rechtsbewusstsein meint eine ,grobe Rechtskenntnis, ein ,Gefühl` dafür, wie die Dinge an diesem Ort und zu dieser Zeit“" (ebd., S. 220) sind und sein sollten. Teilweise wird auch die „Akzeptanz von Rechtsstaatlichkeit“ (ebd.) darunter gefasst. Das individuelle Rechtsbewusstsein wird durch eigene und berichtete positive oder negative Erfahrungen mit Justiz und Recht geprägt, d. h. durch das erlebte Gerechtigkeitsempfinden.

- Rechtskenntnis meint demgegenüber das Wissen über rechtliche Zusammenhänge und Bestände in spezifischen Bereichen, allerdings kein fachmännisches Detailwissen einzelner Paragrafen, sondern die Kenntnis von normativen Leitlinien, mit denen gesellschaftliche Zusammenhänge geregelt sind (ebd., S. 222).

- Unter Anspruchswissen wird schließlich die subjektive Empfindung gefasst, über Rechte zu verfügen und diese individuell beanspruchen und durchsetzen zu können. Kontextbezogen und situativ kann diese Einschätzung unterschiedlich ausfallen, gleich einem Werkzeug eingesetzt werden oder von vornherein als nicht gegeben erachtet werden. Damit sich Anspruchswissen entwickelt, bedarf es einer „Selbstmobilisierung“ (ebd., S. 223): einerseits im ökonomischen Sinne als Kosten-Nutzen-Abwägung, andererseits als Selbstermächtigung im Sinne eines ,Wechsel[s] von einer ausgelieferten zu einer selbstbewussten Position“ (ebd.). Neben der Situation und dem Kontext wird davon ausgegangen, dass (Un-)Gleichheiten und (Un-)Abhängigkeiten auf das Anspruchswissen wirken (ebd.; Blankenburg, 1995, S. 26). 
$\mathrm{Zu}$ den offensichtlichsten objektiven Faktoren der Rechtsmobilisierung werden die vorhandenen juristischen Verfahrensregeln, die (monetären) Kosten und die soziale Einbindung gezählt (Baer, 2017, S. 226 f.). Mit den Verfahrensregeln wird angesprochen, ob etwas überhaupt im Recht thematisiert ist und wie (subjektive) Rechte eingefordert werden können, was also fallbezogen juristisch überhaupt möglich ist (Kocher, 2013, S. 73). Daneben beeinflussen Zeit, Geld und soziale Beziehungen als Mobilisierungsressourcen, wie leicht oder schwer eine Rechtsinanspruchnahme möglich ist. Im Konfliktfall spielt es eine Rolle, welcher Zeiteinsatz vonnöten und möglich ist, ob soziale oder materielle Zugewinne oder etwa Verluste zu erwarten sind, ob es um persönliche oder anonyme Beziehungen geht, ob alleine (isoliert) oder in Gruppenbezügen gehandelt wird, ob es emotionale Verstrickungen oder Abhängigkeiten gibt etc. (Baer, 2017, S. 228 f.). Hierbei lassen sich verschiedene Muster erkennen. Eine Rechtsmobilisierung im Konfliktfall fällt etwa bei vertrauten, langjährigen und andauernden Beziehungskonstellationen schwerer als bei kurzen, unpersönlichen und beendeten Bindungen, wie sie bei Schadensfällen im Straßenverkehr bestehen (ebd., S. 229; Blankenburg, 1995, S. 47).

Potenziell können alle angesprochenen subjektiven und objektiven Faktoren die individuelle oder kollektive Rechtsinanspruchnahme und -durchsetzung fördern oder als Barrieren wirken. Dementsprechend weisen Strategien der Rechtsmobilisierung unterschiedliche Ansatzpunkte auf. Sie reichen von der rechtlichen Wissensvermittlung (z. B. durch Schulungen oder durch die Aufbereitung von Schriftstücken in verständlicher Sprache) über die Einübung, dieses Wissen (diskursiv oder juristisch) anzuwenden, bis zur Rechtsberatung und strategischen Prozessführung (Fuchs, 2019a, S. 243 ff.). Insbesondere die strategische Prozessführung kann ein Mittel sein, um über den Einzelfall hinaus (politische) Aufmerksamkeit auf ein Problem zu lenken, die juristisch „herrschende Meinung“ aufzuweichen oder Rechtskonformität einzufordern (ebd., 2012, S. 53 ff.). Deshalb wird die strategische Prozessführung von Fuchs (2012) als „Partizipationskanal“ bezeichnet.

Somit sind Mobilisierungsprozesse des Rechts mehrdimensional und wie Recht insgesamt stark mit Macht und Herrschaft verquickt. Recht ist „eines der wichtigsten Mittel, um Macht auszuüben und um Macht zu begrenzen“ (Baer, 2017, S. 14). Immanent bleibt dabei ein „Doppelcharakter: Recht kann emanzipatorisch und ermächtigend oder unterdrückend, ausschließend und stigmatisierend sein" (Fuchs, 2019a, S. 243).

Menschen mit Behinderungen sind nun einerseits mit den allgemeinen Barrieren der Rechtsmobilisierung konfrontiert. Nebe und Weber (2014, S. 302 ff.) zeigten z. B. in einer schriftlichen Befragung von rund 3.200 schwerbehinderten Arbeitnehmer*innen mit einer 
Hörschädigung (Stichprobe nach dem Schneeballsystem), dass eine Arbeitsassistenz vor allem dann genutzt wird, wenn die Anspruchsberechtigten über einen guten Kenntnisstand der gesetzlichen Möglichkeiten verfügen. Andererseits gesellen sich für die Personengruppe neben den allgemeinen Barrieren der Rechtsmobilisierung diejenigen der mangelnden Barrierefreiheit hinzu. Diese begründen sich darin, dass Umwelten und Informationen häufig so gestaltet sind, dass mit Beeinträchtigungen eine eigenständige Nutzbarkeit nicht gegeben ist (Kocher, 2013, S. 74). Wie Rambausek (2017, S. 434 ff.) in ihrer rechtssoziologischen Studie zur Inanspruchnahme von Artikel 19 UN-BRK nachzeichnet, sind es folglich kumulative Benachteiligungen, die die Rechtsdurchsetzung von (mobilitätsbeeinträchtigten) Menschen mit Behinderungen erschweren.

\subsection{Zwischenfazit}

Im Kapitel 4 wurden Erkenntnisse und Arbeiten aus der betrieblichen Mitbestimmungsforschung vorgestellt, um zu beleuchten, wie auf dem allgemeinen Arbeitsmarkt in der Privatwirtschaft die Arbeit von Betriebsräten jenseits der formalen Vorgaben ausfällt. Diese Vorgehensweise wurde gewählt, um im weiteren Verlauf der Arbeit auf den Ergebnissen zur betrieblichen Interessenvertretung als potenziellem Referenzrahmen aufbauen zu können.

Zur Einführung der betrieblichen Mitbestimmung in der Privatwirtschaft ist keine „Kontrollinstitution“ (Blankenburg, 1977, S. 49) vorgesehen. Die Rechtsmobilisierung der betrieblichen Mitbestimmung bleibt weitestgehend den Arbeitnehmer*innen und -gebern überlassen. Lediglich in fallbezogenen Konflikten kann das Arbeitsgericht involviert werden (ebd.). Ist ein Betriebsrat im privatwirtschaftlichen Bereich vorhanden, wird dessen Arbeit durch Beziehungen (zum Management, zur Belegschaft und gremienintern), Haltungen und Rollenzuschreibungen beeinflusst. Die innerbetriebliche Position eines Betriebsrats kann von völliger Ignoranz bis zur machtvollen Einflussnahme reichen. Mögliche qualitative Unterschiede sind in der Arbeit von Kotthoff (1981) eindrucksvoll herausgearbeitet. Das gremieninterne Selbstverständnis ist der erste Schritt zur betrieblichen Einflussnahme eines Betriebsrats, so der Forscher. Hierzu müssen Verhältnisse als veränderbar gedeutet werden, wozu äußere Bezugspunkte notwendig sind. Die vorgestellten quantitativen Studien zeigen, dass neben den ,weichen“ Faktoren der Beziehungen und Haltungen die „harten“ Faktoren wie die Branche, die Unternehmensgröße, die Freistellungen der Mitglieder, deren Sach- und Fachkompetenz oder die gewerkschaftliche Einbindung die Rolle des Betriebsrats ausformen. 
Insgesamt verdeutlichen die Studienergebnisse, dass eine proaktive, einflussnehmende Arbeit von Betriebsräten nicht der Regelfall ist, wie dies vergleichende Typologien zeigen ein in der empirischen Mitbestimmungsforschung gängiges Analyseinstrument, um das Agieren von Betriebsräten und daraus resultierende Effekte zu systematisieren und zu kontrastieren. Ein zentraler Untersuchungsaspekt sind ferner Betriebsvereinbarungen. Sie gelten als Indikator für interessenwirksames Handeln und sind in hoher Anzahl besonders häufig bei Betriebsräten gegeben, die als stark eingeschätzt werden. Beide Motive der empirischen Beobachtung von betriebsrätlichem Handeln werden im späteren Forschungsteil dieser Arbeit aufgegriffen: die Typologie als Analyseinstrument und Betriebsvereinbarungen als Unterscheidungsmerkmal für betriebliches Agieren (siehe Abschnitt 7.2.2 Typologie der Werkstatträte zur Annäherung an die Durchsetzungsstärke).

Die abschließende Erörterung von Rechtsmobilisierungsprozessen bestätigt, dass die Inanspruchnahme und die Umsetzung von Recht durch subjektive und objektive Faktoren beeinflusst werden und teilweise sehr voraussetzungsvoll sind. So wirken in der Rechtsmobilisierung von Menschen mit Behinderungen kumulative Benachteiligungen. Dies kann insbesondere für Werkstattbeschäftigte gelten, deren Kapitalarten - um die Argumentationslinie von Rambausek (2017, S. 38 ff.) aufzugreifen - durch die Werkstattbeschäftigung gering ausfallen.

Bezogen auf die empirischen Erkenntnisse zur Interessenvertretung der Beschäftigten in WfbM lässt sich festhalten, dass auf freiwilliger Basis bereits vor Einführung des Rechtsanspruchs auf Mitwirkung in Werkstätten im Jahr 1996 viele Gremien bestanden. Nur wenigen davon gelang es, die Aufgabe einer betrieblichen Interessenvertretung zu erfüllen. In den Studienergebnissen von 1990 wird resümiert, dass ,die Mitwirkung der Behinderten in hohem Maße der Betreuung bedarf. Sie ist auch in den besten Fällen keine autonome Interessenvertretung, deren Durchsetzungskraft aus dem Prozeß der kollektiven Interessenorganisation der Belegschaft hervorgeht“" (Breit \& Kotthoff, 1990, S. 76).

Aus der Zeit nach der Einführung der WMVO im Jahr 2001 liegen zwei quantitative Studien zur Werkstattratsarbeit aus dem (sonder-)pädagogischen Bereich vor (Schlummer, 2004; Hoffmann, 2012). Diese können zeigen, dass Werkstatträte ihre Arbeit aufgenommen haben. Es liegen keine Indizien vor, dass die Gremien formal gar nicht bestehen. In den Studien finden sich wichtige Hinweise zum Mitwirkungsgeschehen nach Einführung der WMVO, wenngleich diese auf einige wenige Erhebungsaspekte zentriert und regional begrenzt bleiben. 
Ein Ausschnitt aus einer kulturanthropologischen Studie (Lahoda, 2018) liefert einen qualitativen Eindruck zur Situation eines Werkstattrats. Hierbei wird ein Bild der Unselbstständigkeit skizziert. Dieser Werkstattrat schafft es nicht, sich vom Fachpersonal zu lösen und ein eigenständiger Ansprechpartner im Werkstattgeschehen zu werden. Jedoch wird er vonseiten der Vertrauensperson in diesem Prozess auch nicht unterstützt. So scheint eine innerbetriebliche Einflussnahme für diesen Werkstattrat sehr weit entfernt.

Aus den praxisbezogenen Beschreibungen zur Arbeit von Werkstatträten geht schließlich hervor, dass die formalen Vorgaben der WMVO nicht gänzlich umgesetzt werden. Prekär ist der Befund, dass ein Fünftel der antwortenden Werkstatträte nach den Befragungsergebnissen von Werkstatträte Deutschland (BVWR, 2013) keine Werkstattversammlungen durchführte, was das formale Mindestmaß des Kontakts zur Wählerbasis der Werkstattbeschäftigten ist.

Insgesamt zeigt sich, dass bisher keine ausreichenden Forschungsergebnisse vorliegen, die belastbare Aussagen über die strukturell verankerten Beteiligungsbemühungen in WfbM im Kontext der aktuellen Entwicklungen erlauben. Diese Beobachtung war der Ausgangspunkt, um mit einer eigenen empirischen Erhebung dem Umsetzungsstand zur Beteiligung in WfbM und der Arbeit von Werkstatträten nachzugehen. 